\title{
Abatacept in rheumatoid arthritis-associated interstitial lung disease: short-term outcomes and predictors of progression
}

\author{
Marika Tardella $^{1}$ D $\cdot$ Marco Di Carlo $^{1}$ D $\cdot$ Marina Carotti $^{2}\left(\mathbb{D} \cdot\right.$ Andrea Giovagnoni $^{2}$ D $\cdot$ Fausto Salaffi $^{1}$ (D)
}

Received: 25 April 2021 / Revised: 27 June 2021 / Accepted: 30 June 2021 / Published online: 27 July 2021

(c) The Author(s) 2021

\begin{abstract}
Introduction Interstitial lung disease in rheumatoid arthritis (RA-ILD) is an extra-articular involvement that impairs the prognosis and for which there is still no well-coded treatment. The aim of this study was to evaluate abatacept (ABA) effectiveness and safety in patients with RA-ILD.

Methods RA-ILD patients who started ABA treatment were consecutively enrolled. Chest high-resolution computed tomography (HRCT), clinical, laboratory and respiratory function variables were collected at baseline and after 18 months of ABA treatment. HRCT abnormalities were evaluated using a computer-aided method (CaM). ABA response was established based on the change in the percentage of fibrosis evaluated at HRCT-CaM, dividing patients into "worsened" (progression $\geq 15 \%$ ), "improved" (reduction $\geq 15 \%$ ), and "stable" (changes within the 15\% range). The multivariate regression model was used to assess the associations between RA characteristics and ABA response.

Results Forty-four patients ( $81 \%$ women, mean age $59.1 \pm 8.0$, mean disease duration of $7.5 \pm 3.1$ years) were studied. Five patients (11.4\%) showed RA-ILD progression, 32 patients (72.6\%) were considered stable, and 7 patients (16.0\%) showed an RA-ILD improvement. The proportion of current smokers was significantly different between "worsened" patients, respect to those defined as "improved/stable" $(p=0.01)$. Current smoking habit $(p=0.005)$ and concomitant methotrexate treatment $(\mathrm{p}=0.0078)$ were the two variables related to RA-ILD progression in multivariate regression analysis.

Conclusion Treatment with ABA is associated with a RA-ILD stability or improvement in the $88.6 \%$ of patients. Current smoking habit and concomitant treatment with methotrexate are the modifiable factors associated with RA-ILD worsening.

\section{Key Points}

- Abatacept plays a favourable role in the control of RA-ILD, with a significant worsening in only $11.4 \%$ of patients during a 18-month followup period.

- The predictive variables related to RA-ILD progression during abatacept therapy are the concomitant treatment with methotrexate and current smoking habit.
\end{abstract}

Keywords Abatacept · High-resolution computed tomography · Interstitial lung disease $\cdot$ Rheumatoid arthritis

Marika Tardella

marikatardella@gmail.com

Marco Di Carlo

dica.marco@yahoo.it

Marina Carotti

marina.carotti@gmail.com

Andrea Giovagnoni

a.giovagnoni@univpm.it

Fausto Salaffi

fausto.salaffi@gmail.com

1 Rheumatology Clinic, Università Politecnica Delle Marche, Via Aldo Moro, 25 - 60035 - Jesi, Ancona, Italy

2 Department of Radiology, Ospedali Riuniti, Università Politecnica Delle Marche, Ancona, Italy

\section{Introduction}

Rheumatoid arthritis (RA) is a progressive systemic autoimmune disorder characterized by articular and extra-articular manifestations affecting about $0.5 \%$ of the adult population in Western countries [1]. Interstitial lung disease (ILD) is one of the most important extra-articular manifestations in RA [2]. The prevalence of RA-ILD varies from 1 to $67 \%$ depending on the method used to assess lung involvement and the study design [3-6]. The most commonly associated risk factors for predicting RA-ILD are advanced age, old age at onset of RA, male gender, smoking status and presence of anti-citrullinated peptide antibodies (ACPA) [7, 8]. In 
addition, some effective drugs used to treat RA can cause lung toxicity [9].

High-resolution computed tomography (HRCT) of chest provides valuable information about ILD, including the pattern and extent of the disease [10]. HRCT abnormalities are found in $48-68 \%$ of asymptomatic patients and $90 \%$ of symptomatic patients with RA [11, 12]. During an average follow-up of 1.5 years, up to $57 \%$ of patients with asymptomatic RA-ILD have experienced a HRCT progression [13]. The usual interstitial pneumonia (UIP) pattern is more frequent in men and is associated with a worse prognosis, while the non-specific interstitial pneumonia (NSIP) pattern is more related to the female gender and has a better prognosis $[14,15]$. The 5 -year survival rate is $36 \%$ in patients with RA-ILD-UIP and $94 \%$ in patients with RA-ILD-NSIP, confirming the favourable outcome of patients with this last pattern [14].

This scenario highlights the need for effective treatment for RA-ILD, but its management is still debated and somewhat controversial [16]. In addition, the pulmonary toxicity of some disease-modifying anti-rheumatic drugs (DMARDs), particularly methotrexate (MTX), is still debated [17]. Immunosuppressive treatments also increase the risk of infection and, in particular, of severe lung infection with a high rate of hospitalization. On the other hand, certain biologic DMARDs (bDMARDs) demonstrated a promising effectiveness in slowing or stopping the progression of RA-ILD. Among these, abatacept (ABA), a T lymphocyte co-stimulation antagonist used in the treatment of RA, has shown some efficacy in the treatment of RA-ILD. $\mathrm{ABA}$ is also promising in light of the reduced infectious risk if compared to other bDMARDs [18]. However, the number of studies published on this issue is still small and mostly retrospective [19-21].

Therefore, the main aim of this study was to evaluate the efficacy and safety of ABA treatment in RA-ILD patients and, as a second aim, to identify predictors of an unfavourable treatment outcome.

\section{Methods}

\section{Study population and assessment}

This study included patients with a diagnosis of RA according to the American College of Rheumatology/European League Against Rheumatism classification criteria [22] and with a coexisting diagnosis of ILD, according to the criteria of the American Thoracic Society/ATS/ERS 2015 [23].

From January 2016 to December 2019, RA-ILD patients attending the outpatient and inpatient clinics of the Rheumatology Clinic of the Polytechnic University of Marche (Italy) were consecutively enrolled. Inclusion criteria were the presence of RA-ILD and the need to start biotechnological drug for active RA, refractory to current therapy, if present. Patients who were concomitantly receiving MTX or other conventional synthetic DMARDs (csDMARDs) and/or glucocorticoids with a dosage of less than $10 \mathrm{mg} /$ day prednisone or equivalent were included. We also included RA patients previously treated with bDMARS, discontinued due to intolerance or ineffectiveness. Patients with a history of pulmonary disease except for ILD, active malignancy, chronic heart failure and previous treatment with ABA were excluded.

ABA treatment was started at a dose of $125 \mathrm{mg} /$ week subcutaneously for all patients, and this time was considered as time zero of the study. Patients were then followed according to normal daily practice with semiannual outpatient visits. A trained nurse monitored the administration of the drug and the occurrence of adverse events weekly by phone or e-mail.

Baseline data were collected by a rheumatologist at time zero and included demographic variables, smoking habits, disease duration (defined as time since RA diagnosis), concomitant therapies and assessment of disease activity (Clinical Disease Activity Index (CDAI) [24] and Health Assessment Questionnaire-Disability Index (HAQ-DI) [25]). The presence of rheumatoid factor (RF) and ACPA was recorded. On the same day, pulmonary symptoms were assessed using the modified Borg Dyspnoea Index (BDI) [26].

Pulmonary function test (PFT), single-breath diffusion lung capacity of carbon monoxide (DLCO, $\%$ predicted, corrected for haemoglobin) and HRCT were performed within 2 weeks of the starting of ABA and after 18 months. Patients who discontinued $\mathrm{ABA}$ due to intolerance or ineffectiveness (CDAI persistently higher than 14) or who did not undergo HRCT after 18 months were excluded from the study. The diagnosis of ILD was performed using chest HRCT, and a quantitative evaluation of pulmonary fibrosis was performed using a computerized method of quantification $(\mathrm{CaM})$, based on what has been described in detail in previous works [27-29]. HRCT images were reconstructed and analysed by OsiriX MD 7, a DICOM visualization software (OsiriX MD version 7, 64-bit format) on a Mac Mini (2.8 GHz Intel Core 2 Duo Desktop Computer, 16 GB random access memory; Apple Computer, Cupertino, CA, USA) with Mac OSX 10.12.2 operating system. Lung parenchymal abnormalities on HRCT were coded and evaluated by two independent radiologists, expert in lung diseases and blinded to the clinical data, using the CaM quantification process. No patient underwent lung biopsy.

HRCT examination was repeated at 18 months after time zero, i.e. initiation of ABA treatment. This examination was also assessed semiquantitatively with CaM.

The local Ethics Committee (Comitato Unico Regionale-ASUR Marche, No 20150458 AS) approved the protocol. The study was conducted in accordance with the Helsinki Declaration in its fifth edition (2000). All patients signed the informed consent. 


\section{Statistical analysis}

Data were recorded in a Microsoft Excel database and processed with MedCalc 19.0.6 (statistical software packages for Windows XP). The Kolmogorov-Smirnov test was used to verify the normal distribution. Where appropriate, the medians and interquartile ranges (IQR), as well as the means and standard deviations (SD), are presented.

A parametric two-sample $t$ test and one-way analysis of variance (ANOVA) test were used to compare continuous variables and the $\chi 2$ test to compare categorical variables between patients. A two-sided coupled t test and the nonparametric Wilcoxon signed rank test were used to compare values at baseline and after 18-months of follow-up.

The analyses of the chest HRCT investigations performed at baseline and after 18 months were conducted dividing patients into three groups on the basis of the CaM-HRCT progression: patients with a lung fibrosis progression $\geq 15 \%$ were defined as "worsened", those with a reduction of $\geq 15 \%$ were defined as "improved", all other patients were defined as "stable". The $15 \% \mathrm{CaM}$ variation threshold resulted from the determination of the standard deviation of the mean value variation after 18 months of follow-up.

Finally, we performed multivariate corrected regression analysis in order to assess the strength of the association between RA characteristics at baseline and HRCT response to ABA. The quantification of $\mathrm{CaM}$ was considered as dependent variable. The covariates included age, sex, disease duration, age at disease onset, smoking habit, RF presence, ACPA presence, CDAI and HAQ-DI. The results were expressed as multivariate regression coefficient $(\mathrm{R})$ and corrected square regression coefficient $\left(\mathrm{R}^{2}\right)$ for the number of variables included in the analysis. This allows to calculate the predictivity of each multivariate model based on the number of variables inserted in the model. The significance has been set to $\mathrm{p}<0.05$.

\section{Results}

Fifty-four patients were included at time zero: $10(18.5 \%)$ patients were eliminated during the course of the study, of whom 4 patients experienced a minor adverse event ( 3 for skin rush and one patient for diarrhoea) and 6 patients for ineffectiveness of ABA after 6 months of treatment (CDAI persistently higher than 22). No severe adverse events or deaths were reported in the followed cohort.

We therefore analysed the data of 44 patients $(81 \%$ women) who completed the study. The mean age was $59.1 \pm 8.0$ years, and the mean disease duration was $7.5 \pm 3.1$ years. Twenty-three (52.3\%) patients were ACPA positive and $28(63.6 \%) \mathrm{RF}$ positive. At baseline the percentage of current smokers was $38.6 \%$. Mean clinical and instrumental data at time zero are summarised in Table 1. All patients were concomitantly treated with csDMARD, in particular MTX (20 patients, 45.5\%), hydroxychloroquine (10 patients, $22.7 \%$ ), leflunomide (8 patients, $18.2 \%$ ), sulfasalazine (6 patients, $13.6 \%$ ) at time zero. Sixteen (36.4\%) patients were previously treated with a bDMARD, including etanercept (6 patients, $13.6 \%$ ), adalimumab (6 patients, $13.6 \%$ ), and tocilizumab (4 patients, $9.2 \%$ ). A total of 31 (70.4\%) patients were treated with corticosteroids at a mean dose of 3.7 (range 1.25-8.5) mg prednisolone/day equivalent. No patients developed tuberculosis during ABA therapy. Four patients underwent prophylactic antituberculous therapy in the month prior to inclusion in the study due to QuantiFERON test positivity.

The patients experienced a significant improvement in RA disease activity and joint function. The mean CDAI score decreased from 34.66 to $10.11(\mathrm{p}<0.001)$, and the mean HAQ-DI score decreased from 1.45 to $0.75(\mathrm{p}<0.001)$ at 18 months (Table 1).

With regard to chest HRCT findings, significant changes in the $\mathrm{CaM}$ score were not detectable in the whole cohort
Table 1 Demographic characteristics, disease activity, functional disability, lung function, and high-resolution computed tomography data at the baseline (T0) and after 18 months of treatment (T18), expressed in means and standard deviations

\begin{tabular}{llllll}
\hline & T0 & \multicolumn{5}{l}{ T18 } \\
\hline & Mean & SD & Mean & SD & $\mathrm{p}^{*}$ \\
Age (years) & 59.05 & 8.03 & - & - & \\
Disease duration (years) & 7.55 & 3.09 & - & - & \\
CDAI & 34.66 & 10.05 & 10.11 & 7.58 & $<0.001$ \\
HAQ-DI & 1.45 & 0.32 & 0.75 & 0.29 & $<0.001$ \\
Borg Dyspnea Index & 2.54 & 1.23 & 1.90 & 1.01 & 0.01 \\
DLco (\% predicted) & 58.69 & 8.24 & 61.26 & 11.23 & 0.22 \\
FVC (\% predicted) & 82.29 & 4.86 & 81.24 & 11.97 & 0.59 \\
HRCT-CaM fibrosis (percentage) & 19.41 & 5.89 & 18.94 & 6.06 & 0.71 \\
\hline
\end{tabular}

Legend and abbreviations: * =two-sided paired Student $\mathrm{t}$ test; $\mathrm{SD}=$ standard deviation; $\mathrm{CDAI}=\mathrm{Clinical}$ Disease Activity Index; HAQ-DI=Health Assessment Questionnaire Disability Index; DLco=diffusion lung capacity of carbon monoxide; $\mathrm{FVC}=$ forced vital capacity; $\mathrm{HRCT}=$ high-resolution computed tomography; $\mathrm{CaM}=$ computer-aided method. 
$(\mathrm{p}=0.71)$. At the end of the 18 months follow-up, $5(11.4 \%)$ patients showed a HRCT deterioration of RA-ILD, 32 (72.6\%) were considered stable, and $7(16.0 \%)$ patients showed an HRCT improvement. Figure 1 shows an example of an improved patient. Analysing the differences between groups, the proportions of current smokers and of patients treated with MTX were significantly higher in the "worsened" compared to those defined as "improved/stable" (80\% vs 33.35\%, $\mathrm{p}=0.01$ and $60 \%$ vs $38.6 \%, \mathrm{p}=0.01$, respectively).

At the multivariate regression analysis, the predictive variables related to RA-ILD progression during ABA therapy were concomitant treatment with MTX $(p=0.0078)$ and current smoking habit $(p=0.0054)$. Gender, disease duration, ACPA presence, RF presence, DLco, FVC, CDAI and HAQDI were not significantly associated with RA-ILD worsening (Table 2).

\section{Discussion}

In this study it has been demonstrated that ABA plays a favourable role in the control of RA-ILD, with a significant worsening in only $11.4 \%$ of patients during the 18 -month follow-up period.

This study consolidates some research already conducted regarding the value of ABA in RA-ILD. The first study on the safety of ABA in RA-ILD described four patients with RA-ILD exacerbated or manifested for the first time during treatment with csDMARDs. The patients were treated with ABA for a mean period of 35 months, without showing a worsening of lung function [20]. Nakashita and colleagues investigated the effect of ABA and other bDMARDs in two retrospective studies of RA-ILD patients [30, 31]. In the first study, they evaluated a group of patients starting therapy with bDMARDs and divided them into two groups according to the presence or absence of ILD at HRCT screening of the chest. After 12 months, patients who developed exacerbations or new ILD were all on TNFi therapy, whereas patients treated with ABA or tocilizumab had no exacerbations or onset of ILD in either group [30]. In the second study, patients with RA-ILD were evaluated, 16 of whom were starting ABA treatment and 46 of whom were starting TNFi treatment. After 12 months, ABA-treated patients showed no worsening of RA-ILD at chest HRCT, whereas $30 \%$ of TNFi-treated patients revealed a worsening of RA-ILD [31].

A Spanish multicentre retrospective study examined 63 RA-ILD patients, 15 of whom developed ILD immediately after the introduction of csDMARDs or bDMARDs. The authors investigated HRCT findings at baseline and after 12 months only in patients with persistent dyspnoea (22 patients, 34\%). Of these, 50\% showed stabilisation, 36.4\% improvement and $13.6 \%$ worsening of ILD [19]. The same Spanish group recently expanded the multicentre study including 263 RA-ILD patients treated with ABA [21]. After 12 months of treatment, only 3 of 67 asymptomatic patients at baseline had mild dyspnoea, while $20 \%$ showed improvement in dyspnoea. FVC remained stable or improved in $87.7 \%$ of patients and DLCO in $90.6 \%$ of patients. HRCT improved in 24 cases $(18.8 \%)$, while it worsened in 30 (23.4\%) and the rest of patients remained stable. They also found a corticosteroid-sparing effect of ABA therapy.

This study is currently the one with the largest number of RA-ILD patients enrolled and treated with ABA. The obtained results show a clear majority of patients who "remain stable" or "improve" compared to those who "worsen", thus affirming that ABA is a safe treatment in RA-ILD patients. Kurata and colleagues showed also that, in a RA population, after the initiation of bDMARD therapy, pre-existing airway disease is an independent risk factor for the onset or exacerbation of ILD, whereas ABA therapy is a protective factor [32]. ABA has a low-risk of worsening pre-existing ILD and could therefore play an important role in the clinical management of RA-ILD patients. We could speculate that ABA has a "protective effect" on the onset/ exacerbation of ILD because, on one hand, the efficacy of blocking T-cell co-stimulation in the non-infectious lung inflammatory process has been demonstrated in the animal
Fig. 1 High-resolution computed tomography scans of a patient with rheumatoid arthritis-associated interstitial lung disease at baseline, starting abatacept treatment $(\mathbf{A})$, and after 18 months of therapy (B). In $\mathbf{A}$ are detectable "groundglass" opacities (asterisks) and pulmonary consolidations (arrowheads), while in B "ground-glass" opacities are significantly reduced and pulmonary consolidations disappeared
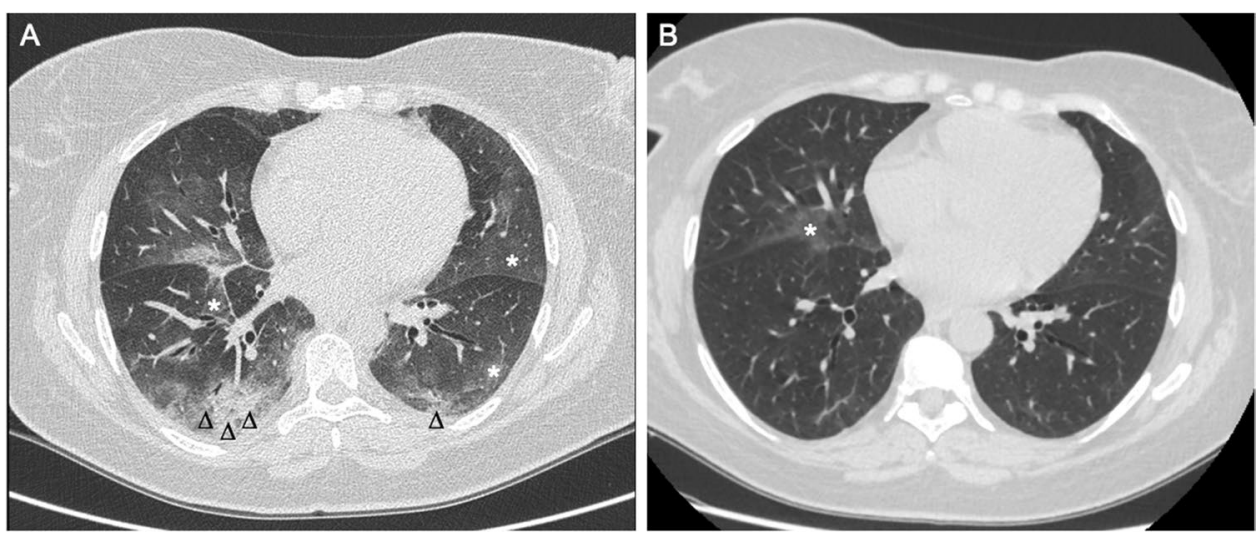
Table 2 Multivariate regression analysis of the variables predictive of pulmonary fibrosis evaluated at high-resolution computed tomography by the computer-aided method (dependent variable)

\begin{tabular}{lcllll}
\hline Independent variables & Coefficient & Standard error & $\mathrm{t}$ & $\mathrm{p}$ & $\mathrm{r}$ partial \\
\hline (Constant) & 1.6370 & & & & \\
Age (years) & -0.0012 & 0.0050 & -0.238 & 0.8131 & -0.0427 \\
Gender & 0.0254 & 0.1084 & 0.235 & 0.8161 & 0.0420 \\
Disease duration (years) & 0.0157 & 0.0176 & 0.891 & 0.3799 & 0.1580 \\
ACPA positivity & -0.0129 & 0.0712 & -0.182 & 0.8566 & -0.0327 \\
RF positivity & 0.0030 & 0.0790 & 0.038 & 0.9694 & 0.0069 \\
Current smokers & -0.3485 & 0.1165 & -2.992 & 0.0054 & -0.4733 \\
Methotrexate use & -0.3956 & 0.1390 & -2.847 & 0.0078 & -0.4552 \\
DLco (\% predicted) & 0.0052 & 0.0061 & 0.848 & 0.4030 & 0.1505 \\
FVC (\% predicted) & -0.0088 & 0.0088 & -1.006 & 0.3224 & -0.1777 \\
CDAI & -0.0074 & 0.0038 & -1.962 & 0.0588 & -0.3324 \\
HAQ-DI & -0.0173 & 0.1459 & -0.119 & 0.9059 & -0.0214 \\
\hline
\end{tabular}

Abbreviations: $\mathrm{ACPA}=$ anti-cytrullinated protein antibodies; $\mathrm{RF}=$ rheumatoid factor; $\mathrm{DLco}=$ diffusion lung capacity of carbon monoxide; $\mathrm{FVC}=$ forced vital capacity; CDAI =Clinical Disease Activity Index; HAQ-DI = Health Assessment Questionnaire Disability Index model of interstitial pneumonia; on the other hand, it is the drug with the lowest infectious risk compared to the other bDMARDs, thus causing a lower incidence of respiratory tract infections $[18,33,34]$. Regarding the latter, it is important to point out that other biotechnology drugs, recommended for RA patients with pre-existing ILD, have a high infectious risk and high rate of neutropenia or reduction of serum immunoglobulins $[35,36]$. In fact, although there are no comparison studies, rituximab and tocilizumab treatments are associated with higher infectious risk than ABA in meta-analyses, and there are reports of exacerbations of persistent ILD or onset of ILD in RA patients on rituximab or tocilizumab therapy $[37,38]$. In addition, further observations should be pointed out if we consider antifibrotic drugs in RA-ILD therapy. There are still some ongoing studies on this topic, but currently available data, extrapolated from some case reports, describe the successful use of nintedanib or pirfenidone in RA-ILD. Therefore, we believe that careful choice of therapy for RA-ILD patients is useful, particularly if a restrictive pulmonary syndrome is already present.

In the case of persistent active synovitis during ABA therapy, we suggest combining one or more DMARDs, preferably hydroxychloroquine or sulfasalazine for their low toxic effects on the lung if there are more than three inflamed joints, while it would be useful to undergo the patient to locoregional infiltrative therapy if there are two or fewer inflamed joints. In case of persistent active disease, it's useful replacing the biotechnological drug as international guidelines suggest. For this reason, patients with high disease activity were excluded from our study.

In our cohort the percentage of "worsened" patients is higher than the evidence in the international literature, and this may be related to the different HRCT assessment method. To our knowledge, this is the first study using HRCT-CaM to estimate response to therapy in patients with
RA-ILD. Recently we demonstrated that a quantitative analysis of ILD using a CaM is more responsive than applying a semi-quantitative visual method in assessing ILD progression in systemic sclerosis [27]. Therefore, the possibility of using standardised CaMs shared by the scientific community for HRCT quantification of ILD could be evaluated, both in research and clinical settings, due to the greater sensitivity in detecting differences [5] and more reliable results.

As a second goal, namely the identification of progression predictors for treatment response, current smokers and MTX-treated patients seemed to respond poorly to ABA. The relationship between cigarette smoking and respiratory disease is well documented. In addition, a strong causal relationship between cigarette smoking, the presence of ACPA and the development of RA-ILD has been widely demonstrated [39]. Therefore, all smoking patients should be helped to stop smoking because of its documented pulmonary toxicity.

In contrast, the relationship between MTX therapy and pulmonary response to ABA is challenging to explain [40]. MTX-induced pulmonary toxicity, presented as acute/subacute or rarely as chronic pneumonia, has been a subject of debate for many years [41, 42]. In recent years, however, this has been questioned with more emphasis on the increased risk of pulmonary infections in patients during MTX therapy rather than direct lung damage. In a meta-analysis of 21 studies from 1990 to 2011, including 8,276 RA patients, it was found that MTX was not associated with an increased risk of total adverse respiratory events and that there were no differences in the risk of pulmonary involvement between patients taking MTX and those not taking it. There was an increased risk of lower respiratory tract infection [43]. In clinical practice, distinguishing MTX-induced pulmonary toxicity from RA-ILD is a challenge and detecting the presence of an infection can be difficult. The worsening of ILD 
detected in the combined ABA and MTX patients in our study is therefore a fact of non-unique interpretation. Mochizuki and coworkers performed a retrospective study of 131 RA-patients treated with ABA and MTX, and they too found MTX to be a negative prognostic factor for lung response to ABA, as the RA-ILD patients who worsened were all on MTX-ABA combination therapy [44]. The most reasonable interpretation in light of recent data is that patients requiring ABA and MTX combination therapy have a more aggressive disease and may have more frequent extra-articular involvement. Although MTX is still the drug of choice for RA therapy, it may be advisable to reduce or discontinue it when clinical remission is achieved in RA-ILD patients, also in the light of international guidelines [45].

This scenario is also complicated by the presence of subclinical ILD in about $30 \%$ of RA patients, evaluated with chest HRCT [46, 47]. The lack of a valid screening tool to detect the presence of ILD in connective tissue diseases is another point of interest, due to the high exposure to ionising radiation of chest HRCT. A good correlation between HRCT and chest ultrasound in ILD detection has been demonstrated $[48,49]$, offering a valid and feasible tool for the detection of pulmonary involvement in RA patients [50], but not yet validated in international studies.

This study has some limitations: firstly, a low number of enrolled patients; secondly, a control group was not recruited; finally, we do not have data on the onset of ILD.

In conclusion, the use of ABA in the treatment of RA-ILD patients can be considered a first choice, especially for its proven safety and therefore for its likely efficacy on lung damage. It can also be stated that MTX therapy should be used with caution in patients with ILD due to the increased risk of infection.

\begin{abstract}
Author contribution FS conceived and designed the study and the protocol. MC and AG performed the HRCT examinations and their relative interpretation and were involved in revising the paper for important intellectual content. FS, MT, and MDC carried out data interpretation and analysis. FS, MT, MDC, and MC wrote the paper. MT, FS and MDC were involved in drafting the article or revising it critically for important intellectual content. All authors approved the final version to be submitted for publication.
\end{abstract}

Funding Open access funding provided by Università Politecnica delle Marche within the CRUI-CARE Agreement.

\section{Compliance with ethical standards}

\section{Disclosure None.}

Open Access This article is licensed under a Creative Commons Attribution 4.0 International License, which permits use, sharing, adaptation, distribution and reproduction in any medium or format, as long as you give appropriate credit to the original author(s) and the source, provide a link to the Creative Commons licence, and indicate if changes were made. The images or other third party material in this article are included in the article's Creative Commons licence, unless indicated otherwise in a credit line to the material. If material is not included in the article's Creative Commons licence and your intended use is not permitted by statutory regulation or exceeds the permitted use, you will need to obtain permission directly from the copyright holder. To view a copy of this licence, visit http://creativecommons.org/licenses/by/4.0/.

\section{References}

1. Salaffi F, De Angelis R, Grassi W (2005) MArche Pain Prevalence INvestigation Group (MAPPING) study. Prevalence of musculoskeletal conditions in an Italian population sample: results of a regional community-based study. I The MAPPING study. Clin Exp Rheumatol 23:819-828

2. Turesson C, O'Fallon WM, Crowson CS, Gabriel SE, Matteson EL (2003) Extra-articular disease manifestations in rheumatoid arthritis: incidence trends and risk factors over 46 years. Ann Rheum Dis 62:722-727

3. Restrepo JF, del Rincón I, Battafarano DF, Haas RW, Doria M, Escalante A (2015) Clinical and laboratory factors associated with interstitial lung disease in rheumatoid arthritis. Clin Rheumatol 34:1529-1536

4. Raimundo K, Solomon JJ, Olson AL et al (2019) Rheumatoid arthritis-interstitial lung disease in the United States: prevalence, incidence, and healthcare costs and mortality. J Rheumatol 46:360-369

5. Salaffi F, Carotti M, Di Carlo M, Tardella M, Giovagnoni A (2019) High resolution computed tomography of the lung in patients with rheumatoid arthritis. Medicine 98(38):e17088

6. Zou YQ, Li YS, Ding XN et al (2012) The clinical significance of HRCT in evaluation of patients with rheumatoid arthritis-associated interstitial lung disease: a report from China. Rheumatol Intern 32:669-673

7. Mori S, Koga Y, Sugimoto M (2012) Different risk factors between interstitial lung disease and airway disease in rheumatoid arthritis. Respir Med 106:1591-1599

8. Bergström U, Jacobsson LT, Nilsson JÅ et al (2011) Pulmonary dysfunction, smoking, socio-economic status and the risk of developing rheumatoid arthritis. Rheumatology (Oxford) 50:2005-2013

9. Hyldgaard C, Hilberg O, Pedersen AB et al (2017) A population-based cohort study of rheumatoid arthritis-associated interstitial lung disease: comorbidity and mortality. Ann Rheum Dis 76:1700-1706

10. Bellia M, Cannizzaro F, Scichilone N et al (2009) HRCT and scleroderma: semi-quantitative evaluation of lung damage and functional abnormalities. Radiol Med 114:190-203

11. Kanat F, Levendoglu F, Teke T (2007) Radiological and functional assessment of pulmonary involvement in the rheumatoid arthritis patients. Rheumatol Int 27:459-466

12. Zrour SH, Touzi M, Bejia I et al (2005) Correlations between high-resolution computer tomography of the chest and clinical function in patient with rheumatoid arthritis. Prospective study in 75 patients. Joint Bone Spine 72:41-47

13. Dawson J, Fewins H, Desmond J, Lynch M, Graham D (2002) Predictors of progression of HRCT diagnosed fibrosing alveolitis in patients with rheumatoid arthritis. Ann Rheum Dis 61:517-521

14. Lee HK, Kim DS, Yoo B et al (2005) Histopathologic pattern and clinical features of rheumatoid arthritis-associated interstitial lung disease. Chest 127:2019-2027

15. Kim EJ, Elicker BM, Maldonado F et al (2010) Usual interstitial pneumonia in rheumatoid arthritis-associated interstitial lung disease. Eur Respir J 35:1322-1328 
16. Mori S (2015) Management of rheumatoid arthritis patients with interstitial lung disease: safety of Biological antirheumatic drugs and assessment of pulmonary Fibrosis. Clin Med Insights Circ Resp Pulm Med 9(Suppl 1):41-49

17. Roubille C, Haraoui B (2014) Interstitial lung diseases induced or exacerbated by DMARDS and biologic agents in rheumatoid arthritis: a systematic literature review. Semin Arthritis Rheum 43:613-626

18. Yun H, Xie F, Delzell E et al (2016) Comparative risk of hospitalized infection associated with biologic agents in rheumatoid arthritis patients enrolled in medicare. Arthtiris Rheumatol 68:56-66

19. Fernández-Díaz C, Loricera j, Castañeda S, et al (2018) Abatacept in patients with rheumatoid arthritis and interstitial lung disease: a national multicenter study of 63 patients. Semin Arthritis Rheum 48:22-27

20. Mera-Varela A, Pérez-Pampín E (2014) Abatacept therapy in rheumatoid arthritis with interstitial lung disease. J Clin Rheumatol 20:445-446

21. Fernández-Díaz C, Castañeda S, Melero-González RB et al (2020) Abatacept in interstitial lung disease associated with rheumatoid arthritis: national multicenter study of 263 patients. Rheumatology (Oxford) 59(12):3906-3916

22. Aletaha D, Neogi T, Silman AJ et al (2010) 2010 Rheumatoid arthritis classification criteria: an American College of Rheumatology/European League Against Rheumatism collaborative initiative. Arthritis Rheum 62:2569-2581

23. Raghu G, Rochwerg B, Zhang Y et al (2015) An official ATS/ ERS/JRS/ALAT clinical practice guideline: treatment of idiopathic pulmonary fibrosis. an update of the 2011 clinical practice guideline. American journal of respiratory and critical care medicine 192:e3-19

24. Aletaha D, Nell VP, Stamm T et al (2005) Acute phase reactants add little to composite disease activity indices for rheumatoid arthritis: validation of a clinical activity score. Arthritis Res Ther 7:R796-806

25. Fries JF, Spitz P, Kraines RG et al (1980) Measurement of patient outcome in arthritis. Arthritis Rheum 23:137-145

26. Borg GA (1982) Psychophysical bases of perceived exertion. Med Sci Sports Exerc 14:377-381

27. Salaffi F, Carotti M, Tardella M et al (2020) Computed tomography assessment of evolution of interstitial lung disease in systemic sclerosis: comparison of two scoring systems. Eur J Intern Med 76:71-75

28. Ariani A, Carotti M, Gutierrez M et al (2014) Utility of an opensource DICOM viewer software (OsiriX) to assess pulmonary fibrosis in systemic sclerosis: preliminary results. Rheumatol Int 34:511-516

29. Salaffi F, Carotti M, Bosello S et al (2015) Computer-aided quantification of interstitial lung disease from high resolution computed tomography images in systemic sclerosis: correlation with visual reader-based score and physiologic tests. Biomed Res Int 2015: 834262.

30. Nakashita T, Ando K, Kaneko N, Takahashi K, Motojima S (2014) Potential risk of TNF inhibitors on the progression of interstitial lung disease in patients with rheumatoid arthritis. BMJ Open 4:e00561.

31. Nakashita T, Ando K, Takahashi K, Motojima S (2016) Possible effect of abatacept on the progression of interstitial lung disease in rheumatoid arthritis patients. Respir Investig 54:376-379

32. Kurata I, Tsuboi H, Terasaki M et al (2019) Effect of biological disease-modifying anti-rheumatic drugs on airway and interstitial lung disease in patients with rheumatoid arthritis. Intern Med 58:1703-1712

33. Harigai M, Ishiguro N, Inokuma S et al (2016) Postmarketing surveillance of the safety and effectiveness of abatacept in
Japanese patients with rheumatoid arthritis. Mod Rheumatol 26:491-498

34. Michot JM, Bigenwald C, Champiat $S$ et al (2016) Immunerelated adverse events with immune checkpoint blockade: a comprehensive review. Eur J Cancer 54:139-148

35. Holroyd CR, Seth R, Bukhari M et al (2019) The British Society for Rheumatology biologic DMARD safety guidelines in inflammatory arthritis. Rheumatology (Oxford) 58(2):e3-e42

36. Manfredi A, Cassone G, Furini F et al (2019) Tocilizumab therapy in rheumatoid arthritis with interstitial lung disease: a multicenter retrospective study. Intern Med J 50(9):1085-1090

37. Zhou T, Shen Q, Peng H et al (2018) Incidence of interstitial pneumonitis in non-Hodgkin's lymphoma patients receiving immunochemotherapy with pegylated liposomal doxorubicin and rituximab. Ann Hematol 97(1):141-147

38. Akiyama M, Kaneko Y, Yamaoka K et al (2016) Association of disease activity with acute exacerbation of interstitial lung disease during tocilizumab treatment in patients with rheumatoid arthritis: a retrospective, case-control study. Rheumatol Int 36(6):881-889

39. Israël-Assayag E, Fournier M, Cormier Y (1999) Blockade of T cell costimulation by CTLA4-Ig inhibits lung inflammation in murine hypersensitivity pneumonitis. J Immunol 163:6794-6799

40. Sandhu A, Dhir V, Ahmad S et al (2020) Clinico-genetic model to predict methotrexate intolerance in rheumatoid arthritis. Clin Rheumatol 39:201-206

41. Salaffi F, Manganelli P, Carotti M, Subiaco S, Lamanna G, Cervini C (1997) Methotrexate-induced pneumonitis in patients with rheumatoid arthritis and psoriatic arthritis: report of five cases and review of the literature. Clin Rheumatol 16:296-304

42. Khadadah ME, Jayakrishnan B, Al-Gorair S et al (2002) Effect of methotrexate on pulmonary function in patients with rheumatoid arthritis - a prospective study. Rheumatol Int 22:204-207

43. Conway R, Low C, Coughlan RJ, O’Donnell MJ, Carey JJ (2014) Methotrexate and lung disease in rheumatoid arthritis: a metaanalysis of randomized controlled trials. Arthritis Rheumatol 66:803-812

44. Mochizuki T, Ikari K, Yano K et al (2018) Long-term deterioration of interstitial lung disease in patients with rheumatoid arthritis treated with abatacept. Modern Rheumatology 29(3):413-417

45. Singh JA, Saag KG, Bridges SL Jr, Akl EA, Bannuru RR, Sullivan MC et al (2016) American College of Rheumatology guideline for the treatment of rheumatoid arthritis. Arthritis Care Res (Hoboken) $68: 1-25$

46. Olson AL, Swigris JJ, Sprunger DB et al (2011) Rheumatoid arthritis-interstitial lung disease-associated mortality. Am J Respir Crit Care Med 183:372-378

47. Delle Sedie A, Doveri M, Frassi F et al (2010) Ultrasound lung comets in systemic sclerosis: a useful tool to detect lung interstitial fibrosis. Clin Exp Rheumatol 28(5 Suppl 62):S54

48. Tardella M, Gutierrez M, Salaffi F et al (2012) Ultrasound in the assessment of pulmonary fibrosis in connective tissue disorders: correlation with high- resolution computed tomography. J Rheumatol 39:1641-1647

49. Tardella M, Di Carlo M, Carotti M, Filippucci E, Grassi W, Salaffi F (2018) Ultrasound B-lines in the evaluation of interstitial lung disease in patients with systemic sclerosis. Medicine 97:e0566

50. Di Carlo M, Tardella M, Filippucci E, Carotti M, Salaffi F (2021) Lung ultrasound in patients with rheumatoid arthritis: definition of significant interstitial lung disease. Clin Exp Rheumatol PMID: 33938789

Publisher's note Springer Nature remains neutral with regard to jurisdictional claims in published maps and institutional affiliations. 